\title{
The diagnosis of pancreatic mucinous cystic neoplasm and associated adenocarcinoma in males: An eight-institution study of 349 patients over 15 years
}

\author{
Cecilia G. Ethun MD ${ }^{1}$ | Lauren M. Postlewait MD ${ }^{1}$ | Mia R. Mclnnis BA ${ }^{1}$ | \\ Nipun Merchant MD $^{2,3}$ | Alexander Parikh $M^{3}$ | Kamran Idrees $M^{3}$ | \\ Chelsea A. Isom MD ${ }^{3}$ | William Hawkins $M^{4}$ | Ryan C. Fields $M^{4}$ | \\ Matthew Strand MD ${ }^{4}$ | Sharon M. Weber MD ${ }^{5}$ | Clifford S. Cho MD ${ }^{5,6}$ | \\ Ahmed Salem MD ${ }^{5}$ | Robert C.G. Martin MD ${ }^{7}$ | Charles R. Scoggins MD \\ David Bentrem MD ${ }^{8}$ | Hong J. Kim MD ${ }^{9}$ | Jacquelyn Carr MD ${ }^{9}$ | \\ Syed A. Ahmad MD ${ }^{10}$ | Daniel E. Abbott MD ${ }^{5,10}$ | Gregory Wilson MD ${ }^{10}$ | \\ David A. Kooby MD ${ }^{1}$ | Shishir K. Maithel MD ${ }^{1}$
}

\footnotetext{
${ }^{1}$ Division of Surgical Oncology, Department of Surgery, Winship Cancer Institute, Emory University, Atlanta, Georgia

${ }^{2}$ Division of Surgical Oncology, Department of Surgery, University of Miami, Miami, Florida

${ }^{3}$ Division of Surgical Oncology, Department of Surgery, Vanderbilt University Medical Center, Nashville, Tennessee

${ }^{4}$ Department of Surgery, Washington University School of Medicine, St Louis, Missouri

5 Department of Surgery, University of Wisconsin School of Medicine and Public Health, Madison, Wisconsin

${ }^{6}$ Department of Surgery, University of Michigan, Ann Arbor, Michigan

7 Division of Surgical Oncology, Department of Surgery, University of Louisville, Louisville, Kentucky

${ }^{8}$ Department of Surgery, Northwestern University Feinberg School of Medicine, Chicago, Illinois

${ }^{9}$ Division of Surgical Oncology, Department of Surgery, University of North Carolina, Chapel Hill, North Carolina

${ }^{10}$ Division of Surgical Oncology, Department of Surgery, University of Cincinnati Cancer Institute, Cincinnati, Ohio

Correspondence

Shishir K. Maithel, MD, 1365C Clifton Rd. NE, Building C, 2nd floor, Atlanta, GA 30322.

Email: smaithe@emory.edu
}

BACKGROUND: Per WHO, 2000 classification, pancreatic mucinous cystic neoplasms (MCN) are defined by presence of ovarian stroma, and are primarily located in the pancreatic body/tail of females. The incidence of MCN and associated malignancy in males, since, standardization of MCN diagnostic-criteria is unknown.

METHODS: MCN resections from 2000 to 2014 at eight institutions of the Central-PancreasConsortium were included, and divided into early (2000-2007) and late (2008-2014) timeperiods. Primary aim was to characterize $\mathrm{MCN}$ and associated adenocarcinoma/high-gradedysplasia (AC/HGD) in males versus females over time.

RESULTS: Of 1667 resections for pancreatic cystic lesions, 349 pts (21\%) had MCNs: 310 (89\%) female, 39 (11\%) male. Patients were equally divided between early $(n=173)$ and late $(n=176)$ time-periods. $M C N$ in male-patients decreased over time (early: $15 \%$, late: $7 \% ; P=0.036$ ), as did pancreatic head/neck location (early: $22 \%$, late: $11 \% ; P=0.01$ ). $M C N$-associated $A C / H G D$ was more frequent in males versus females (39 vs. $12 \% ; P<0.001$ ). The overall rate of $\mathrm{MCN}$ associated $\mathrm{AC} / \mathrm{HGD}$ remained stable (early: $17 \%$, late: $13 \% ; P=0.4$ ), and was identical in males (39\%) over both time-periods. Males with AC/HGD had more LN-positive disease versus females (57 vs. $22 \% ; P=0.039$ ).

CONCLUSIONS: As the diagnostic-criteria of MCN have standardized over time, $\mathrm{MCN}$ diagnosis has decreased in males and head/neck location. Despite this, $\mathrm{MCN}$-associated adenocarcinoma/high-grade dysplasia has been stable and remains high in males. Any male with suspected $\mathrm{MCN}$, regardless of location, should undergo resection.

KEYWORDS

high-grade dysplasia, ovarian stroma, surgical resection 


\section{1 | INTRODUCTION}

Cystic neoplasms of the pancreas are a rare and assorted group of pancreatic lesions that display a variety of clinical and pathologic characteristics, and carry a range of malignant potential. They represent approximately $10 \%$ of all pancreatic neoplasms, but account for nearly one third of all pancreatic resections. ${ }^{1}$ In addition to serous cystadenomas and intraductal papillary mucinous neoplasms (IPMNs), mucinous cystic neoplasms (MCNs) are one of the most common cystic neoplasms of the pancreas. ${ }^{2,3}$

Current guidelines recommend complete resection of all suspected MCNs due to the risk of associated high-grade dysplasia and invasive adenocarcinoma, which ranges between $10 \%$ to as high as $46 \%$ in the literature. $^{3-9}$ Thus, achieving an accurate diagnosis of $\mathrm{MCN}$ is paramount, yet can be difficult due to the often overlapping clinical, radiographic, cytologic, and histopathologic features with branch-duct IPMNs. In 2000, as part of an effort to better differentiate MCN from IPMN, the World Health Organization $(\mathrm{WHO})$ defined $\mathrm{MCN}$ by the presence of ovarian stroma on histopathology. ${ }^{10}$ While this histologic distinction has helped to standardize the pathologic diagnosis of MCN, the clinical, radiographic, and endoscopic characteristics of these lesions remain important preoperative diagnostic tools for determining management strategy.

One of the most striking clinical features unique to MCNs is that they occur almost exclusively in the pancreatic body and tail of premenopausal women., ${ }^{4,7}$ Still, based on the 2000 WHO criteria, MCNs do occur in males, and male gender has been associated with a higher malignant potential, yet these patients are a group that has largely been unstudied. ${ }^{8,11-13}$ The purpose of this study was utilize a large, multi-institutional database to characterize MCNs and assess the incidence of associated adenocarcinoma/high-grade dysplasia (AC/HGD) in males compared to females over time.

\section{2 | METHODS}

The Central Pancreas Consortium is a collaboration of eight U.S. academic institutions: Emory University, Northwestern University, University of Cincinnati, University of Louisville, University of North Carolina, University of Wisconsin, Vanderbilt University, and Washington University in St. Louis. All patients who underwent resection of a pancreatic cystic neoplasm from 2000 to 2014 were assessed. Only patients with pathologically confirmed MCN were included in analysis. Patients with other pancreatic cystic neoplasms were excluded. Pertinent baseline demographic, perioperative, and pathologic data were reviewed retrospectively using patient charts. Pathologic review was performed by experienced $\mathrm{Gl}$ pathologists at each institution at the time of resection. Institutional Review Board approval was obtained at each institution prior to data collection.

The primary objective was to compare the clinical, radiographic, and pathologic characteristics of MCNs in male versus female patients. The secondary objectives were to compare the incidence of $\mathrm{MCN}$ s diagnosed in male patients, located in the head and neck of the pancreas, and associated with AC/HGD over two time periods: early (2000-2007) and late (2008-2014). These time periods were chosen to ensure equal cohort size. In addition, based on the well-known observation that it takes several years for published data and guidelines to be widely adopted into clinical practice, ${ }^{14}$ the authors believed that 8 years was an appropriate timeframe to assess changes in diagnostic patterns.

Statistical analyses were conducted using SPSS 22.0 software (IBM Inc., Armonk, NY). Male and female patients who underwent pancreatic resection for $\mathrm{MCN}$ were compared using chi-squared analysis for categorical variables, and Student's $t$-test for continuous variables. Statistical significance was predefined as two-tailed $P<0.05$.

\section{3 | RESULTS}

Of 1667 patients who underwent resection of a pancreatic cystic neoplasm, 349 (21\%) had MCNs. The remaining cystic neoplasms that were excluded from analysis were: IPMN $(n=586)$, serous cystadenoma ( $n=227)$, cystic adenocarcinoma $(n=132)$, pseudocyst $(n=122)$, pseudopapillary tumor $(n=86)$, and other $(n=165)$.

Of the $349 \mathrm{MCN}$ patients, 310 (89\%) were female and 39 (11\%) were male. Clinicopathologic comparisons between male and female patients are shown in Table 1. Male patients were older than female patients ( $65 \mathrm{yrs}$ vs. $52 \mathrm{yrs} ; P<0.001$ ), and were more likely to present with jaundice (11 vs. $1 \%$; $P=0.001$ ), have lesions located in the head of the pancreas (49 vs. $11 \%$; $P<0.001$ ), and display pancreatic ductal dilatation on pre-operative imaging (42 vs. $14 \%$; $P<0.001$ ). Male patients also more frequently underwent pancreaticoduodenectomy compared to female patients ( 46 vs. $10 \%$; $P<0.001$ ), although distal pancreatectomy was still the most common procedure performed in both groups. Adenocarcinoma and/or high-grade dysplasia was identified within surgical specimens in $39 \%$ of male patients and $12 \%$ of female patients $(P<0.001)$. Lymph node positive disease was identified in $57 \%$ of male patients and $22 \%$ of female patients $(P=0.04$ ).

Patients were divided by surgery date into early (2000-2007; $n=173,50 \%)$ and late (2008-2014; $n=176,50 \%)$ time periods (Fig. 1). There was a decrease from the Early to Late time periods in the proportion of $\mathrm{MCN}$ patients who were male ( $15 \mathrm{vs} .7 \% ; P=0.04$ ) and of $\mathrm{MCNs}$ located in the pancreatic head/neck (22 vs. $11 \%$; $P=0.01$ ). There was no difference in the proportion of $\mathrm{MCNs}$ associated with $\mathrm{AC} / \mathrm{HGD}$ between the early and late groups (17 vs. $13 \% ; P=0.41)$. On stratumspecific analysis by gender, the proportion of male $\mathrm{MCN}$ patients with associated AC/HGD remained stable at $39 \%$ over both time periods.

\section{4 | DISCUSSION}

Mucinous cystic neoplasms are one of the most common types of cystic neoplasms of the pancreas, and are primarily found in the pancreatic body and tail of premenopausal women. ${ }^{1,2}$ Although uncommon, MCNs are still reported in males, even using modern diagnostic criteria, yet few studies focus on this rare and unique group of patients. ${ }^{11-13}$ In this study, we utilized a large, multi-institutional database to investigate the clinicopathologic characteristics of MCNs diagnosed in male compared to female patients, and assessed 
TABLE 1 Clinicopathologic characteristics of male versus female patients with mucinous cystic neoplasms

\begin{tabular}{|c|c|c|c|}
\hline Variable & $\begin{array}{l}\text { Female } \\
(n=310 \\
89 \%)\end{array}$ & $\begin{array}{l}\text { Male } \\
(n=39, \\
11 \%)\end{array}$ & $\begin{array}{l}P \text { - } \\
\text { value }\end{array}$ \\
\hline Age (yrs), mean $\pm S D$ & $52 \pm 14$ & $65 \pm 12$ & $<0.001$ \\
\hline $\mathrm{BMI}\left(\mathrm{kg} / \mathrm{m}^{2}\right)$, mean $\pm \mathrm{SD}$ & $29 \pm 7$ & $27 \pm 8$ & 0.35 \\
\hline \multicolumn{4}{|l|}{ Race, $n$ (\%) } \\
\hline White & $241(78)$ & $35(90)$ & 0.46 \\
\hline African-American & $53(17)$ & $4(10)$ & \\
\hline Latino & $5(2)$ & $0(0)$ & \\
\hline Other & $11(3)$ & $0(0)$ & \\
\hline \multicolumn{4}{|l|}{ ASA class, $n(\%)$} \\
\hline 1 & $68(24)$ & $9(24)$ & 0.31 \\
\hline 2 & $132(46)$ & $12(32)$ & \\
\hline 3 & $81(28)$ & $16(43)$ & \\
\hline 4 & $6(2)$ & $0(0)$ & \\
\hline \multicolumn{4}{|l|}{ Presentation, n (\%) } \\
\hline Asymptomatic & $108(35)$ & $14(37)$ & 0.001 \\
\hline Jaundice & $3(1)$ & $4(11)$ & \\
\hline Pain & $147(48)$ & $11(29)$ & \\
\hline Pancreatitis & $33(11)$ & $6(16)$ & \\
\hline Other & $15(6)$ & $3(8)$ & \\
\hline
\end{tabular}

Radiographic appearance, $n$

(\%)

\begin{tabular}{|c|c|c|c|}
\hline Location (head/neck) & $33(11)$ & $18(49)$ & $<0.001$ \\
\hline Septations & $106(40)$ & $8(25)$ & 0.14 \\
\hline Solid component & $51(20)$ & $4(14)$ & 0.62 \\
\hline Mural nodule & $23(9)$ & $5(17)$ & 0.29 \\
\hline Ductal dilatation & $36(14)$ & $14(42)$ & $<0.001$ \\
\hline Tumor size $(\mathrm{cm})$, mean \pm SD & $5.1 \pm 4.2$ & $3.5 \pm 2.7$ & 0.04 \\
\hline \multicolumn{4}{|l|}{ Resection, $n$ (\%) } \\
\hline Distal pancreatectomy & $268(87)$ & $21(54)$ & $<0.001$ \\
\hline Pancreaticoduodenectomy & $32(10)$ & $18(46)$ & \\
\hline Central pancreatectomy & $6(2)$ & $0(0)$ & \\
\hline Total pancreatectomy & $4(1)$ & $0(0)$ & \\
\hline \multicolumn{4}{|l|}{ Technique, $n(\%)$} \\
\hline Open & $193(62)$ & $34(87)$ & 0.004 \\
\hline Minimally invasive & $117(38)$ & $5(13)$ & \\
\hline $\begin{array}{l}\text { Estimated blood loss }(\mathrm{mL}) \\
\text { mean } \pm \mathrm{SD}\end{array}$ & $297 \pm 318$ & $\begin{array}{l}494 \\
\pm 424\end{array}$ & 0.001 \\
\hline $\begin{array}{l}\text { Length of stay (days), mean } \\
\quad \pm \text { SD }\end{array}$ & $7.0 \pm 4.6$ & $\begin{array}{l}11.9 \\
\pm 13.5\end{array}$ & 0.002 \\
\hline Tumor size $(\mathrm{cm})$, mean \pm SD & $5.2 \pm 4.5$ & $3.7 \pm 2.6$ & 0.004 \\
\hline $\begin{array}{l}\text { Adenocarcinoma or high- } \\
\text { grade dysplasia, } n(\%)\end{array}$ & $37(12)$ & $15(39)$ & $<0.001$ \\
\hline \multicolumn{4}{|l|}{ Margin, $n(\%)$} \\
\hline RO & $293(98)$ & $36(92)$ & 0.10 \\
\hline R1 & $7(2)$ & $3(8)$ & \\
\hline Lymph node positive, $n$ (\%) & $6(22)$ & $8(57)$ & 0.04 \\
\hline
\end{tabular}

BMI, body mass index; ASA, American Society of Anesthesiology.

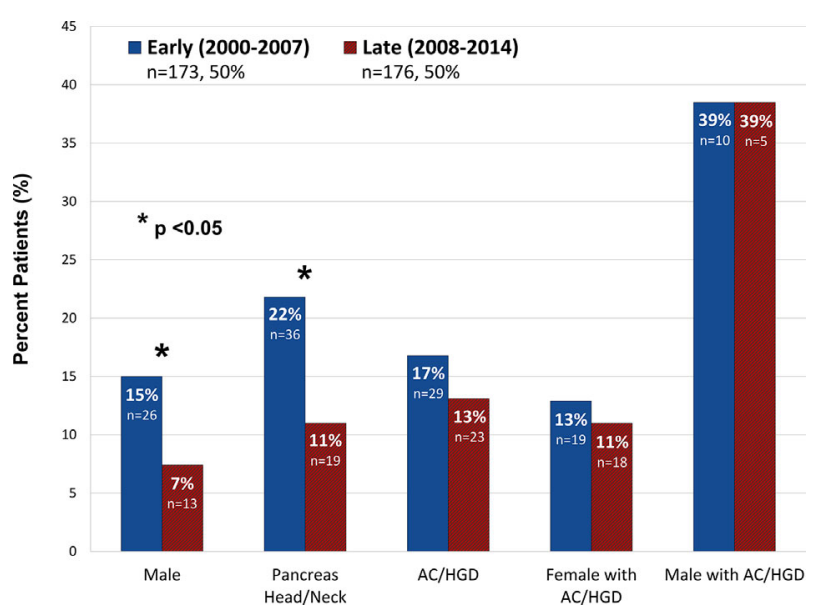

FIGURE 1 Comparison of gender, lesion location, and malignant potential over two time periods: early (2000-2007) and late (2008-2014)

diagnostic trends over time. We found that male patients were more likely to present with obstructing head/neck lesions and have associated AC/HGD compared to females. While the proportion of male MCN patients and lesions located in the pancreatic head/neck decreased over time, the incidence of AC/HGD remained stable, and was particularly high among male patients.

Because of the relatively high risk of associated $A C / H G D$, complete surgical resection is recommended for all suspected MCNs. ${ }^{4}$ Thus, distinguishing MCNs from branch-duct IPMNs, which carry a lower malignant potential and do not always necessitate surgical excision, is important. One of the most striking differences between the two lesions is that the overwhelming majority of MCNs occur in the pancreatic body and tail of female patients, while branchduct IPMNs occur more commonly in the pancreatic head and neck with a nearly even gender distribution. ${ }^{4,7}$ Since 2000 , when the WHO proposed the presence of ovarian stroma as the defining diagnostic criterion for $\mathrm{MCN}$, the association between $\mathrm{MCN}$ and female gender has become even stronger. In a systematic review of 25 studies published from 1996 to 2005 , Goh et al. ${ }^{15}$ found that in studies in which the presence of ovarian stroma was not mandatory for $\mathrm{MCN}$ diagnosis, 80\% (range, 8-100\%) were female patients. In studies, in which the presence of ovarian stroma was mandatory for $\mathrm{MCN}$ diagnosis, however, 99.7\% (range, 98-100\%) were female.

This finding has led some to adopt the belief that MCNs are exclusive to only female patients, and any male patient with an MCN diagnosis likely has a misclassified IPMN. Yet the continued emergence of modern case reports and case series of male patients with histologically confirmed MCN based on the presence of ovarian stroma, demonstrates that, while rare, this population does, in fact, exist. ${ }^{11-13,16,17}$ Although the specific pathogenesis of MCNs is unclear, it is believed to be hormonally mediated, and in a study by Regi et al., ${ }^{16}$ nearly $50 \%$ of the male patients with histologically confirmed MCN were found to have hormonal and/or sexual dysfunction. Furthermore, MCNs in male patients are of particular importance due to their increased malignant potential. As the current authors demonstrated in a previous study, ${ }^{8}$ male gender was independently associated with the presence of $\mathrm{AC} / \mathrm{HGD}$ on pathology, even when taking into account patient comorbidities and other diagnostic features. 
Of the 349 patients with MCNs in the current study, 310 (89\%) were female and 39 (11\%) were male. Male patients were more likely to have obstructing lesions in the pancreatic head/neck, and have associated AC/ HGD compared to female patients. Understanding that this study was performed based on retrospective chart review without centralized pathologic re-review, it is possible that some of these $\mathrm{MCNs}$, particularly in male patients and those diagnosed early on, may have been misclassified branch-duct IPMNs. Allowing 8 years for the $2000 \mathrm{WHO}$ guidelines to integrate into clinical practice, we divided the cohort into early (2000-2007) and late (2008-2014) groups. As expected, the incidence of male gender and lesions located in the pancreatic head/neck each decreased by $50 \%$ from the early to late time periods. Furthermore, the incidence of male gender in the late time period (7\%) fell more closely in line with the incidence quoted in most modern studies (5\%). Importantly, however, there was no change in the incidence of associated $\mathrm{AC} / \mathrm{HGD}$ overall between the time periods, and it remained alarmingly high specifically in male patients (39\%) in both the early and late groups. It is unclear whether this observation represents distinct pathogenesis or more aggressive tumor biology in male compared to female patients with MCN. Regardless, taking a pragmatic approach, this study demonstrates that, even in a modern era nearly a decade after the WHO criteria were published, male patients are still being diagnosed with $\mathrm{MCN}$ and are at high risk for AC/HGD. Thus, any male patient with suspected $\mathrm{MCN}$, regardless of location, should undergo resection.

There are several limitations to this study. As previously mentioned, the retrospective nature of the study makes it challenging to draw definitive conclusions. In addition, without centralized pathologic rereview, histologic confirmation of MCN could not be made. However, this study includes data from seven diverse, academic institutions across the U.S., which eliminates single-institution bias and more closely represents the disease characteristics and general practice patterns of this country. In addition, all involved academic centers have experienced $\mathrm{Gl}$ pathologist who performed the initial pathologic review.

\section{5 | CONCLUSIONS}

In conclusion, as the diagnostic-criteria of mucinous cystic neoplasms (MCN) have standardized over time, MCN diagnosis has decreased in male patients and in the pancreatic head/neck. Despite this, $\mathrm{MCN}$-associated adenocarcinoma/high-grade dysplasia has been stable and remains extremely high in male patients, even in the modern era. Thus, any male patient with suspected $\mathrm{MCN}$, with or without pre-operative histologic confirmation and regardless of location, should undergo resection.

\section{CONFLICTS OF INTEREST}

Nothing to disclose.

\section{REFERENCES}

1. Brugge WR, Lauwers GY, Sahani D, et al. Cystic neoplasms of the pancreas. N Eng J Med. 2004;351:1218-1226.
2. Adsay NV. Cystic neoplasia of the pancreas: pathology and biology. J Gastrointestin Surg. 2008;12:401-404.

3. Brugge WR, Lewandrowski K, Lee-Lewandrowski E, et al. Diagnosis of pancreatic cystic neoplasms: a report of the cooperative pancreatic cyst study. Gastroenterology. 2004;126:1330-1336.

4. Tanaka M, Fernandez-del Castillo C, Adsay V, et al. International consensus guidelines 2012 for the management of IPMN and MCN of the pancreas. Pancreatology. 2012;12:183-197.

5. Crippa S, Salvia R, Warshaw AL, et al. Mucinous cystic neoplasm of the pancreas is not an aggressive entity: lessons from 163 resected patients. Ann Surg. 2008;247:571-579.

6. Reddy RP, Smyrk TC, Zapiach M, et al. Pancreatic mucinous cystic neoplasm defined by ovarian stroma: demographics, clinical features, and prevalence of cancer. Clin Gastroenterol Hepatol. 2004; 2:1026-1031.

7. Crippa S, Fernandez-Del Castillo C, Salvia R, et al. Mucin-producing neoplasms of the pancreas: an analysis of distinguishing clinical and epidemiologic characteristics. Clin Gastroenterol Hepatol. 2010;8: 213-219.

8. Postlewait LM, Ethun CG, McInnis MR, et al. Association of preoperative risk factors with malignancy in pancreatic mucinous cystic neoplasms: a multicenter study. JAMA Surg. 2017;152:19-25.

9. Tanaka M, Chari S, Adsay V, et al. International consensus guidelines for management of intraductal papillary mucinous neoplasms and mucinous cystic neoplasms of the pancreas. Pancreatology. 2006; 6:17-32.

10. Aaltonen LA, Hamilton SR, World Health Organization, International Agency for Research on Cancer. Pathology and genetics of tumours of the digestive system. Lyon, Oxford: IARC Press; Oxford University Press; 2000.

11. Casadei R, Pezzilli R, Calculli L, et al. Pancreatic mucinous cystic neoplasm in a male patient. JOP. 2012;13:687-689.

12. Suzuki M, Fujita N, Onodera $\mathrm{H}$, et al. Mucinous cystic neoplasm in a young male patient. J Gastroenterol. 2005;40:1070-1074.

13. Tokuyama Y, Osada S, Sanada Y, et al. Mucinous cystic neoplasm of the pancreas in a male patient. Rare Tumors. 2011;3: 14.

14. Balas EA. From appropriate care to evidence-based medicine. Pediatr Ann. 1998;27:581-584

15. Goh BK, Tan YM, Chung YF, et al. A review of mucinous cystic neoplasms of the pancreas defined by ovarian-type stroma: clinicopathological features of 344 patients. World J Surg. 2006; 30:2236-2245.

16. Regi P, Salvia R, Cena C, et al. Cystic "feminine" pancreatic neoplasms in men. Do any clinical alterations correlate with these uncommon entities? Int J Surg. 2013;11:157-160.

17. Wouters K, Ectors N. Van Steenbergen W, et al. A pancreatic mucinous cystadenoma in a man with mesenchymal stroma, expressing oestrogen and progesterone receptors. Virchows Archiv. 1998;432:187-189.

How to cite this article: Ethun CG, Postlewait LM, Mclnnis $M R$, et al. The diagnosis of pancreatic mucinous cystic neoplasm and associated adenocarcinoma in males: An eightinstitution study of 349 patients over 15 years. J Surg Oncol. 2017;115:784-787. https://doi.org/10.1002/jso.24582 